\title{
Was ist und was soll Internationale Politische Theorie?
}

\begin{abstract}
Die Internationale Politische Theorie (IPT) analysiert politische Verhältnisse, Strukturen und Institutionen jenseits des Staates. Insbesondere fragt sie nach gerechtfertigten sozialen und politischen Verhältnissen in jenem Raum, d.h. nicht nur nach diesbezüglichen Normen, sondern auch nach den Orten und Modi der Rechtfertigung derselben. Dabei zielt sie ein »Überlegungsgleichgewicht «zwischen deskriptiven und normativen Perspektiven an. Insbesondere bedarf sie einer realistischen Einschätzung der jeweiligen Verhältnisse von Herrschaft und Beherrschung, womit sie den Rahmen des klassischen "Realismus" übersteigt. Die Besonderheit eines »anderen Realismus « zeigt sich an der Vermeidung kurzschlüssiger Verbindungen von Empirie und Normativität. So wird eine kritische politische Theorie möglich.
\end{abstract}

\section{Normative Ordnungen jenseits des Staates}

Wenn es gestattet ist, eine partikulare Entwicklung als Zeichen eines allgemeinen wissenschaftlichen Trends zu deuten, so sehe ich es nicht als zufällig an, dass es im Rahmen des Frankfurter Exzellenzclusters »Die Herausbildung normativer Ordnungen $\aleph^{1}$ zu einer engen Kooperation zwischen Politischer Theorie/Philosophie und den Internationalen Beziehungen gekommen ist ${ }^{2}$ und neben anderen die erste Professur in Deutschland mit dem Titel »Internationale Politische Theorie« eingerichtet wurde. Es mag Frankfurt-spezifisch sein, wie hier die normative Dimension der Herausbildung neuer - insbesondere: trans-, inter- und supranationaler - Ordnungen untersucht wird, doch liegt es insgesamt gesehen auf der Hand, dass die Welt, in der wir leben, und die zu erforschen Aufgabe der Wissenschaften ist, es erfordert, diese Prozesse der Ordnungsbildung, ihre Formen, Chancen, Risiken und Verwerfungen aus einer normativen Perspektive in den Blick zu nehmen. Diese Prozesse werden nicht nur undurchsichtig bleiben, wenn wir die evaluative Binnenperspektive der Beteiligten nicht erschließen; mehr noch, wir werden uns in diesen Analysen selbst nicht finden, wenn wir nicht eine Sprache für das Besondere normativer Ordnungen und Konflikte entwickeln. Das alte Begriffspaar »Erklären« und »Verstehen« kommt als Trias ge-

1 Siehe ausführlicher Normative Orders (2010).

2 Das zeigt auch die von den DVPW-Sektionen »Politische Theorie und Ideengeschichte« und »Internationale Politik« gemeinsam veranstaltete Tagung »Internationale Politische Theorie«, die vom 10. bis 12. Juni 2010 in Frankfurt am Main stattgefunden hat und aus der die Beiträge dieses Symposiums hervorgegangen sind. Mein besonderer Dank gilt in diesem Zusammenhang Nicole Deitelhoff und Peter Niesen sowie Benjamin Herborth und Stefan Gosepath. 
meinsam mit »Kritik« hier zur Geltung, ${ }^{3}$ und so kommt es darauf an, soziale und politische Ordnungen, auch und gerade solche jenseits des Staates, als »Rechtfertigungsordnungen« zu begreifen (vgl. Forst/Günther 2010). In diesem Zusammenhang spielt die Internationale Politische Theorie (IPT) eine wichtige Rolle.

Der IPT geht es, allgemein gesagt, darum, über politische Verhältnisse, Strukturen und Institutionen jenseits des Staates nachzudenken und für sie Begriffe und Bewertungen zu finden. Im Näheren fragt sie nach gerechtfertigten sozialen und politischen Verhältnissen im überstaatlichen Raum, d.h. nicht nur nach den diesbezüglichen Normen, sondern insbesondere - reflexiv gesprochen - nach den Orten und den Modi der Rechtfertigung derselben. Denn diese Orte und Modi bilden sich erst heraus (oder sollten es), und so hat die IPT neben einer rekonstruktiven eine wichtige konstruktive Aufgabe; sie muss in Prozessen denken und dabei normative Maßstäbe für ebendiese finden. Die bestehenden Verhältnisse sind ebenso zu analysieren wie mögliche Formen und Ordnungen der Rechtfertigung, die diese Verhältnisse in legitime bzw. gerechte verwandelten. Es handelt sich also um eine reflexiv gewendete, dabei aber zugleich klassische Fragestellung der politischen Theorie, denn von Dantes Monarchia über Kants Friedensschrift bis zu Helds Vorschlägen zu einer kosmopolitischen Demokratie reicht der Bogen von Theorien über die gerechtfertigte Politik jenseits des Staates. Globalisierungsschübe und entsprechende Theorien gab es in Zeiten des militärischen, religiösen oder wirtschaftlichen Ausgreifens von Gesellschaften in Richtung anderer Gebiete oder gar Erdteile schon immer; und stets waren diese Reflexionen - z.B. als Legitimationen der Kolonisierung oder als Formen ihrer Kritik mit Wertungen verbunden. Wir haben es nicht mit einem gänzlich neuen Feld der politischen Theorie zu tun, wohl aber einem besonders aktuellen, da sich das politische Terrain des Globus angesichts übergreifender Krisen und Kriege immer mehr verengt; es reicht heute bis in die Details der nationalen und regionalen Politik hinein. Und es verlangt, das Paradigma der internationalen Beziehungen (im engeren Sinne) zu überschreiten, da dieses die Komplexität der innerstaatlichen wie auch der transnationalen Realitäten nicht mehr einfangen kann.

Es überrascht daher nicht, dass heute verstärkt Internationale Politische Theorie betrieben wird. Dabei sind zwei aktuelle Tendenzen bemerkenswert. Erstens ist innerhalb der politischen Theorie, und dies ist zweifelsohne dem Einfluss der Rawlsschen Theorie zu verdanken, eine größere und fruchtbare Nähe zwischen Philosophie und Politischer Theorie zu verzeichnen, was sowohl den normativen als auch den institutionentheoretischen Überlegungen zugutekommt; und zweitens kommt es, wie bereits bemerkt, zu einem besseren Austausch zwischen der Politischen Theorie und den Internationalen Beziehungen. Dies ist von Bedeutung, denn die Analyse der Prozesse, Institutionen und Konflikte um eine »neue Weltordnung«, was auch immer das genau heißt, braucht die Expertise beider. Die Zusammenarbeit der »neuen Internationalen Beziehungen« (Hellmann et al. 2003) mit der Internationalen Politischen Theorie sehe ich als ebenso nötig wie vielversprechend an, auch wenn sich in der

3 Es ist in diesem Zusammenhang an der Zeit, auch die klassische Diskussion von »Hermeneutik und Ideologiekritik« neu zu lesen: Karl-Otto Apel u.a. (1971). 
diesbezüglichen Begriffswelt noch einiges finden muss. Vielleicht wird man sagen können, dass Charles Beitz' Klassiker von 1979, Political Theory and International Relations, der den Spannungsbogen zwischen einem Hobbesianischen Realismus und einem Rawlsschen Konstruktivismus öffnete, der Beginn einer Periode der Zusammenarbeit dieser Subdisziplinen war, die immer intensiver wird. Freilich muss man dabei wissen, dass die Differenz zwischen normativen und deskriptiven Ansätzen nicht verschwinden wird; sie muss nur recht genutzt werden. ${ }^{4}$ Es gilt, dass wir auf ein »Überlegungsgleichgewicht« zwischen beiden Perspektiven hinarbeiten müssen (Forst 2007: 345); ${ }^{5}$ nur so kann eine im doppelten Sinne kritische wissenschaftliche Sicht auf unsere Welt gelingen: kritisch darüber aufgeklärt, was ist, und normativ diesbezüglich orientiert, was sein sollte (wenn auch darüber streitend).

\section{Ein anderer Realismus}

Damit dies gelingt, benötigen wir einen realistischen Ansatz. Dies zu hören wird die, die eine an Hobbes angelehnte bestimmte Perspektive auf die internationale Welt »realistisch« nennen, zunächst überraschen, aber das nur, weil sie sich eine sehr spezifische Sicht darauf zurechtgelegt haben, was die Wirklichkeit ist und von welchen Gesetzen sie bestimmt wird; an vielen Stellen schlägt dieser Realismus erkennbar ins Normative um. Die eigentlich realistische Theorie freilich ist in meinen Augen die, die in ihren normativen Weichenstellungen von einer möglichst klaren Analyse der politischen und sozialen bzw. ökonomischen Verhältnisse ausgeht, die wir jenseits der Staaten antreffen - nämlich im Wesentlichen asymmetrische Beziehungen und Strukturen unterschiedlichster Art, mit ganz unterschiedlichen Akteuren auf verschiedensten Ebenen, d.h. ein Komplex von Macht, Herrschaft und Beherrschung, der wahrhaft eine Herausforderung für jede sozialwissenschaftliche Analyse darstellt. ${ }^{6}$ Wer über legitime oder gerechte transnationale Herrschaft nachdenken will, und das ist eine der zentralen Aufgaben der Internationalen Politischen Theorie, muss wissen, welche Herrschafts- und Beherrschungsverhältnisse sich in der Welt, in der wir leben, herausgebildet haben. Dort liegt der Einsatzpunkt einer kritischen politischen Theorie, hier und jetzt - ebenso kontextgebunden wie kontexttranszendierend (Forst 2007: 357-380). Für sie ist transnationale Herrschaft nicht weniger »real« als die Kritik daran und die Frage, was hier Gerechtigkeit hieße.

Dieses Programm kann ich hier nicht weiter entwickeln. Ich will stattdessen den Fokus etwas verengen und - vorrangig ex negativo - auf einige Probleme hinweisen, die es zu vermeiden gilt, wenn man das oben erwähnte Überlegungsgleichgewicht anstrebt und eine kurzschlüssige Verbindung von empirischen und normativen Ansätzen vermeiden will.

4 Siehe dazu insbesondere Zürn (2010).

5 Der Terminus stammt ursprünglich von John Rawls, der ihn allerdings anders verwendete.

6 Zur Differenzierung von Macht, Herrschaft und Beherrschung vgl. Forst (2010b). 


\subsection{Die Idee eines »overlapping consensus»}

Wie viele der Ideen und Begriffe, die Rawls entwickelt hat, ist auch der (nicht elegant $\mathrm{zu}$ übersetzende) des »overlapping consensus« bestechend. Er sollte bei Rawls freilich nicht dazu dienen, wie heute oft angenommen wird, die Prinzipien einer Konzeption der Gerechtigkeit in einer pluralistischen Gesellschaft zu begründen; vielmehr war er dafür gedacht, die Stabilität einer wohlgeordneten und pluralistischen Gesellschaft vorstellbar zu machen (Rawls 1993: Lecture 4). Die zunächst unabhängig begründeten Prinzipien sollen diesem Gedanken zufolge von den Bürgerinnen und Bürgern aus unterschiedlichen Gründen heraus - in Übereinstimmung mit ihren »comprehensive doctrines « - akzeptiert werden. So kann die politische Konzeption der Gerechtigkeit nach Rawls Stabilität gewinnen.

In vielen neueren Diskussionen wird dieses Modell hingegen als solches der $\mathrm{Be}$ gründung von Grundsätzen transnationaler Gerechtigkeit oder der Menschenrechte gedeutet (kritisch dazu Forst 2010a). Die Intention dahinter ist verständlich - man will den »fact of pluralism « unterschiedlicher Kulturen und Religionen berücksichtigen und ethnozentrische Begründungen vermeiden. Daher begibt man sich auf die Suche nach zwischen Kulturen und Gesellschaften in der Weise teilbaren Werten oder Prinzipien, dass sie aus ganz unterschiedlichen Perspektiven stammen und doch an einem bestimmten Punkt überlappen. Diesen Punk markiert dann eine hinreichend »minimale« Menschenrechtsdefinition oder eine des menschlichen Wohlergehens.

Dabei bleibt nicht nur begrifflich unklar, was für eine Art Konsens es ist, wenn Werte geteilt werden, ihre Begründungen jedoch nicht. Ersichtlicherweise kann das gar keine Begründung von irgendetwas sein, nur ein Überlappen eben. Aber wie stellt man diese Überlappungen eigentlich fest? Handelt es sich um einen empirischen Konsens, und wenn ja, einen existierenden oder einen, der erst herzustellen wäre oder doch um einen fiktiven? Aber ein fiktiver Konsens ist nichts als Fiktion. Man kann ihn sich in interkulturellen Diskursen, die man sich erdenkt, erfinden, aber wer spricht da gedanklich mit, und wer ist ausgeschlossen? Und wer hat in diesem Raum der Gründe Autorität? Es scheint, als wäre jeweils schon normativ vorausgesetzt, was erst zu begründen wäre.

Aber mehr noch, wie plausibel ist es eigentlich, einen erdachten oder gar realen universalen Schnittmengenkonsens darüber befinden zu lassen, welche Menschenrechte gelten? Wer wäre etwa auf die Idee gekommen, die »Droits de L'homme et du citoyen« durch einen »overlapping consensus« der damaligen Zeit begründen zu lassen - oder die Allgemeine Erklärung der Menschenrechte in den vierziger Jahren? Und wer würde auf den Gedanken kommen, den Protestierenden auf dem Platz des Himmlischen Friedens oder denen, die in Nigeria die Hungerlöhne eines Ölmultis anklagen, oder den Mönchen in Burma zu sagen, sie mögen doch bedenken, dass Ihre Gerechtigkeits- und Menschenrechtsforderungen über die minimale Schnittmenge eines interkulturellen »overlapping consensus« hinausgehen? Hier droht die Gefahr, normatives Denken gegen diplomatische Überlegungen einzutauschen. Um nicht missverstanden zu werden: Wir brauchen politische Diplomatie, aber zunächst müs- 
sen wir unbefangen fragen, was Menschenrechte oder transnationale Gerechtigkeit bedeuten.

Der Umschlag des Realismus ins Affirmative ist die Gefahr, um die es dabei geht: Man will auf realistische Weise vom Faktum des Pluralismus ausgehen und endet in der Sprachlosigkeit gegenüber der wirklichen Realität, nämlich der von sozialen Konflikten und Kämpfen mit starken moralischen Ansprüchen - die nicht »der Westen« erhebt oder begründet, sondern die Betroffenen selbst, die gegen eine existierende normative Ordnung aufbegehren. Übersieht man das, kann sich auch die Ethnozentrismuskritik, die von unterschiedlicher Seite, nicht nur von postkolonialen Theorien, zu Recht vorgebracht wird, in ihr Gegenteil verkehren - in die Idee, Menschenrechte etwa seien eine rein westliche Idee und Besitz des Westens (oder gar des christlichen Westens). Damit reserviert man diese normative Grammatik des Protests für bestimmte Gesellschaften und übersieht ihre kontextübergreifende Genese und Geltung. Man bürgert zudem die Protestierenden in nichtwestlichen Gesellschaften, die Menschenrechte einfordern, ideell aus diesen aus. Das Resultat ist eine Reifizierung von Kulturen, die falsch ist.

Richtig gesehen, sind bestimmte soziale Konflikte die Geburtsstätte der Menschenrechte-solche, in denen Subjekte ihre Regierung bzw. Aspekte ihres Regiertwerdens als grundsätzlich nicht legitimierbar zurückweisen. Sie klagen konkrete Freiheiten oder Güter ein, auf jeden Fall die Freiheit von bestimmten Gängelungen, aber sie haben dabei in der Regel nichts »Westliches« im Sinn (da westliche Länder nicht selten Teil des Problems sind). Auf jeden Fall klagen sie das Recht ein, nicht unter Normen und Verhältnissen leben zu müssen, die ihnen gegenüber nicht angemessen gerechtfertigt werden können - ihr Recht auf Rechtfertigung, wie ich das nenne. ${ }^{7}$ Von diesem Grundanspruch aus ist eine kritische Theorie gerechtfertigter Verhältnisse zu entwickeln, die Paternalismus und Ethnozentrismus wahrhaft vermeidet - aber auch nicht die höchst reale Perspektive derer übersieht, die um ihr Recht kämpfen, nicht länger als Rechtfertigungsnull angesehen und ignoriert zu werden. Diese Theorie wird an erster Stelle eine Kritik der Rechtfertigungsverhältnisse sein (Forst 2011).

\subsection{Die Funktion der Menschenrechte}

Im gegenwärtigen Diskurs über die Menschenrechte, und zwar in der Politischen Theorie wie auch in den Internationalen Beziehungen, findet sich ein weiteres Verfehlen der rechten Bestimmung von empirischer und normativer Theorie, die dazu führt, dass eine berechtigte Intention in einen falschen Gedanken umschlägt. Es wird dabei ein zu enger Zusammenhang zwischen normativen Überlegungen und institutionellen Konsequenzen hergestellt, mit reduktionistischen normativen Implikationen.

7 Vgl. dazu Forst (2007). Zur Genese von Grundrechten aus Konflikten heraus siehe Forst (2003). 
Dabei wird die Funktion der Menschenrechte, wie etwa Beitz (2009) es in seinem neuen Buch tut, ${ }^{8}$ hauptsächlich darin gesehen, die interne und externe Souveränität von Staaten zu begrenzen - im Letztfall eine Intervention zu rechtfertigen. Dies ist aus der Perspektive der internationalen Politik und des internationalen Rechts eine treffende Funktionsbestimmung, allein, es wird daraus ein falscher normativer Schluss gezogen. Denn es wird angenommen, eine extensive Auslegung und Definition der Menschenrechte könnte Interventionen (von interessierter Seite, wie es nur allzu oft vorkommt) Tür und Tor öffnen. Das Ergebnis ist eine reduzierte Kernliste der Menschenrechte. Eine umfassendere wird als praktisch nicht geboten angesehen, weil sie missbrauchsanfällig ist; sie gilt zudem als nicht legitimerweise durchsetzbar.

Diese Position vermischt freilich unterschiedliche Fragen - die Frage nach den Menschenrechten und der Legitimität von Staaten aus der Sicht ihrer Bürger und Bürgerinnen und, demgegenüber, die Frage nach der externen Souveränität eines Staates und nach Gründen für eine legitime Intervention von außen. Aber so verbunden diese Fragen sind, sind sie zunächst getrennt zu beantworten: Umfang und Begründung der Menschenrechte sind eine Sache, die Frage, wer wann zu einer Intervention legitimiert ist, eine andere.

Bei der Bestimmung der Funktion der Menschenrechte gilt es, sie in ihrem ursprünglichen politischen und sozialen Kontext zu sehen - einer politischen Gemeinschaft, in der um die Grundbedingungen einer gerechtfertigten Grundstruktur gerungen wird und Betroffene bestimmte Rechte fordern, von denen sie glauben, dass niemand sie mit guten Gründen zurückweisen kann. Welche Rechte und welche Gründe dies sind, muss sich in einem Diskurs unter Freien und Gleichen rechtfertigen lassen - womit impliziert ist, dass die Rechte, die diesen Status begründen, vorrangiger Natur sind. Ohne darauf näher eingehen zu können, erhellt daraus, dass die wesentliche Funktion der Menschenrechte zunächst einmal innerhalb einer Gesellschaft bestimmt werden muss - sie gewährleisten dort den Grundstatus Freier und Gleicher, den niemand anderen Menschen verweigern darf. Dieser Grundstatus sichert die Stellung der Einzelnen als vollwertige Mitglieder einer politischen Gemeinschaft. So lässt sich auch ein Menschenrecht auf demokratische Partizipation begründen, wie es in der Allgemeinen Erklärung etwa enthalten ist - und wie es in minimalistischen Theorien der Menschenrechte infrage gestellt wird.

Der menschenrechtliche Anspruch auf demokratische Partizipation, der jeweils von innen erhoben wird, darf somit weder mit dem Verweis auf seine kulturelle Nichtverallgemeinerbarkeit (s.o.) noch mit dem auf die interventionistischen Implikationen solcher Bestimmungen normativ infrage gestellt werden. Im internationalen System sind ohne Zweifel nichtliberale und nichtdemokratische Gesellschaften als Mitglieder zu tolerieren, aber das heißt nicht, dass diese damit Recht hätten, ihren Mitgliedern das Recht auf Demokratie zu verweigern; die Perspektive und Ansprüche der Betroffenen sind für eine normative Betrachtung zentral. Und es heißt auch nicht, dass demokratische Staaten bei einer solchen Verweigerung per se irgendein Recht auf In-

8 Zur folgenden Kritik wiederum Forst (2010a). 
tervention hätten. Die Fälle gerechtfertigter Interventionen, die es gibt, müssen eigens betrachtet und Kriterien hierfür, substantielle und prozedurale, festgelegt werden.

Es ist ein Fehler der Internationalen Politischen Theorie, eine globale Weltschiedsrichterhaltung einzunehmen, die - um es übertrieben auszudrücken - Menschenrechte im Sinne minimaler Kompromissformeln verstünde und eine zu enge Verbindung zwischen der Geltung der Menschenrechte und der Legitimation von Interventionen herstellte. Das hieße, die Realität der Kämpfe um Menschenrechte in verschiedenen Staaten nicht angemessen in den Blick zu nehmen - die Perspektive der Betroffenen, die weder weiter von ihrer Regierung gegängelt noch von westlichen Staaten »befreit « werden wollen.

\subsection{Politik und Ethik}

Ein spiegelbildliches Problem stellt sich dort, wo Internationale Politische Theorie lediglich als eine Form der »angewandten Ethik « betrachtet wird, die den besonderen Sinn des Politischen verkennt. Sie beginnt - etwa als »ideale Theorie « - nicht bei realen Herrschaftsverhältnissen und den konkreten Ansprüchen auf Rechtfertigung in diesen Verhältnissen, sondern mit abstrakten Annahmen über menschliche Bedürfnisse, über Wohlergehen und eine entsprechende Güterversorgung und skizziert dann einen weitgehend abstrakten Kosmopolitismus, der der praktischen Welt fremd gegenübersteht. Die rechte Verteilung funktioniert wie eine Maschine, die die richtigen Ergebnisse produziert, und politische Autonomie - staatlich wie persönlich ist eine nur sekundäre Angelegenheit (Forst 2009).

Auch hier ist ein Realitätsverlust zu beklagen. Die Gerechtigkeit kommt ja als Göttin Justitia nicht in eine Tabula rasa-Welt, sondern in eine konkrete Wirklichkeit sozialer Verhältnisse der Beherrschung und Ausbeutung, und an diesen muss sie ansetzen. Sie operiert zwar mit allgemeinen Prinzipien, doch lokalisiert sie diese in den Kontexten, in denen Gerechtigkeitsansprüche als gezielte und gerichtete erhoben werden. Die Gerechtigkeit muss die Verhältnisse der Beherrschung und der Willkür identifizieren, die $\mathrm{zu}$ »richten « sind.

Ein Verschleifen der Differenz zwischen einer allgemeinen Moral menschlicher Solidarität und einer politischen Moral der Gerechtigkeit, wie bei Peter Singer (2010) etwa, führt zudem dazu, dass die Frage der Verantwortlichkeiten für die Herstellung gerechter Verhältnisse in den Hintergrund gedrängt wird. Wer allein an der Behebung von Mangelzuständen interessiert ist, dem sind die Opfer einer Naturkatastrophe gleich denen, die das Opfer von Ausbeutung und Unterdrückung sind. Ihnen muss auch beiden geholfen werden, aber aus verschiedenen Gründen und auf Weisen, die die Verantwortlichkeiten berücksichtigen. Die Gerechtigkeit zielt auf soziale und politische Verhältnisse, nicht auf Zustände.

So gilt es, eine »geerdete« Konzeption der Gerechtigkeit jenseits des Staates zu entwickeln, die auf der Basis einer umfassenden Kritik transnationaler Herrschaftsund Beherrschungsverhältnisse zu einer normativen und institutionellen Theorie der transnationalen wie auch nationalen Grundstrukturen voranschreitet, die das Recht 
auf Rechtfertigung ins Werk setzen können. Politische und soziale Gerechtigkeit, recht verstanden, erfordert zuallererst eine Grundstruktur der Rechtfertigung als fundamentale Forderung, da es ihr darauf ankommt, Verhältnisse der Beherrschung politisch in solche der Selbstbestimmung umzuwandeln. Und so wie die Verhältnisse der Beherrschung sich nicht in das Schema Nationalstaat versus Weltstaat pressen lassen, so tun es auch die institutionellen Implikationen der Gerechtigkeit nicht. Diese Implikationen auf der Basis einer Rekonstruktion der jeweiligen Unterwerfungsverhältnisse zu extrapolieren ist eine konstruktive Aufgabe der IPT. Sie ist sich dabei aber dessen bewusst, dass politische Konstruktion letztlich eine politische Aufgabe der Praxis ist.

Daher ist die IPT dort am realistischsten, wo sie empirisch fundiert die Verhältnisse benennt, die nicht rechtfertigbar sind und Rechtfertigung als Praxis verhindern. Sie lässt sich auf keine schale Differenz von Kontextualismus und Universalismus ein, weil sie weiß, dass die konkreten Ansprüche der Betroffenen oft die universalistischsten sind, zumindest einen solchen Kern haben. Angesichts solcher Kämpfe um normative Ordnungen steht die politische Theorie nicht abseits, sei es als Bestandsaufnahme eines »overlapping consensus«, als diplomatisches Weltenlenkertum oder abstrakte Idealtheorie, sondern versteht sich selbst als Teil der sozialen Welt. Ihre Objektivität ist eine Stellung nehmende, denn auch sie steht in Kontexten der Rechtfertigung, theoretischen wie praktischen. Eine kritische politische Theorie verhält sich dazu.

\section{Literatur}

Apel, Karl-Otto (Hrsg.) 1971: Hermeneutik und Ideologiekritik. Mit Beiträgen von Karl-Otto Apel, Claus v. Bormann, Rüdiger Bubner, Hans-Georg Gadamer, Hans Joachim Giegel, Jürgen Habermas, Frankfurt a. M.

Beitz, Charles R. 1979: Political Theory and International Relations, Princeton, NJ.

Beitz, Charles R. 2009: The Idea of Human Rights, Oxford.

Forst, Rainer 2003: Toleranz im Konflikt. Geschichte, Gehalt und Gegenwart eines umstrittenen Begriffs, Frankfurt a. M.

Forst, Rainer 2007: Das Recht auf Rechtfertigung. Elemente einer konstruktivistischen Theorie der Gerechtigkeit, Frankfurt a. M.

Forst, Rainer 2009: Zwei Bilder der Gerechtigkeit, in: Forst, Rainer/Hartmann, Martin/Jaeggi, Rahel/Saar, Martin (Hrsg.): Sozialphilosophie und Kritik, Frankfurt a. M., 205-228.

Forst, Rainer 2010a: The Justification of Human Rights and the Basic Right to Justification: A Reflexive Approach, in: Ethics 120: 4, 711-740.

Forst, Rainer 2010b: Noumenal Power (Vortrag auf der Jahrestagung der American Political Science Association (APSA), 2.-5. September 2010, Washington, D.C.), unv. Ms.

Forst, Rainer/Günther, Klaus 2010: Die Herausbildung normativer Ordnungen. Zur Idee eines interdisziplinären Forschungsprogramms, in: Forst, Rainer/Günther, Klaus (Hrsg.): Die Herausbildung normativer Ordnungen. Interdisziplinäre Perspektiven, Frankfurt a. M., i.E.

Forst, Rainer 2011: Kritik der Rechtfertigungsverhältnisse. Perspektiven einer kritischen Theorie der Politik, Berlin, i.E.

Hellmann, Gunther/Wolf, Klaus D./Zürn, Michael (Hrsg.) 2003: Die neuen Internationalen Beziehungen. Forschungsstand und Perspektiven in Deutschland, Baden-Baden.

Normative Orders 2010: Webseite, in: http://www.normativeorders.net; 18.10.2010. 
Rawls, John 1993: Political Liberalism, New York, NY.

Singer, Peter 2010: Leben retten. Wie sich Armut abschaffen lässt - und warum wir es nicht tun, Zürich.

Zürn, Michael 2010: Normativität und Deskription in der Internationalen Politischen Theorie (Beitrag zur Tagung »Internationale Politische Theorie«, 10.-12. Juni 2010, Frankfurt am Main), unv. Ms. 